\title{
White Matter Integrity Reductions in Intermittent Explosive Disorder
}

\author{
Royce Lee ${ }^{*, 1}$, Konstantinos Arfanakis ${ }^{2}$, Arnold M Evia ${ }^{2}$, Jennifer Fanning', Sarah Keedy' and Emil F Coccaro' \\ 'Clinical Neuroscience Research Unit, Department of Psychiatry and Behavioral Neuroscience, School of Medicine, University of Chicago, Chicago, \\ IL, USA; ${ }^{2}$ Department of Biomedical Engineering, Illinois Institute of Technology, Chicago, IL, USA
}

\begin{abstract}
Intermittent explosive disorder (IED), as described in DSM-5, is the categorical expression of pathological impulsive aggression. Previous work has identified neurobiological correlates of the disorder in patterns of frontal-limbic brain activity and dysregulation of serotonergic neurotransmission. Given the importance of short- and-long range white matter connections of the brain in social and emotional behavior, studies of white matter connectivity in impulsive aggression are warranted. Diffusion tensor imaging (DTI) studies in the related conditions of antisocial and borderline personality disorder have produced preliminary evidence of disturbed white matter connectivity in these disorders, but to date there have been no DTI studies in IED. A total of I 32 male and female adults between the ages of I 8 and 55 years underwent Turboprop-DTI on a 3-Tesla MRI scanner. Of these, 42 subjects had IED, 40 were normal controls, and 50 were clinical psychiatric controls with psychiatric disorders without IED. All subjects were free of alcohol, psychotropic medications, or drugs of abuse. The diffusion tensor was calculated in each voxel and maps of fractional anisotropy (FA) were generated. Tract-based spatial statistics (TBSS) were used to compare FA along the white matter skeleton among the three subject groups. IED was associated with lower FA in two clusters located in the superior longitudinal fasciculus (SLF) when compared with the psychiatric and healthy controls. Impulsive aggression and borderline personality disorder, but not psychopathy or antisocial personality disorder, was associated with lower FA in the two clusters within the SLF. In conclusion, IED was associated with lower white matter integrity in long-range connections between the frontal and temporoparietal regions.
\end{abstract}

Neuropsychopharmacology (2016) 4I, 2697-2703; doi:I 0.1038/npp.2016.74; published online 22 June 2016

\section{INTRODUCTION}

Pathological impulsive aggression is characterized in DSM-5 as intermittent explosive disorder (IED). IED is not rare, with a prevalence of 3-5\% (Coccaro, 2012) and a mean age of onset of 14 years (Kessler et al, 2006). Impulsive aggression is operationalized as reactive verbal or physical aggression against people, objects, and animals. Episodes are generally triggered by social provocation and are associated with anger (Coccaro et al, 2014). This is in contrast to instrumental aggression, as seen in psychopathy, that is aggression carried out in pursuit of a reward or dominance (Glenn and Raine, 2009). Current therapeutic approaches such as SSRI medications and cognitive behavioral therapy (CBT) can help, but are not always effective (respectively Coccaro et al, 2009; McCloskey et al, 2008). Thus, there is a real need for new approaches in the clinic. Identifying the neurobiological abnormality causing impulsive aggression would be

*Correspondence: Dr R Lee, Clinical Neuroscience Research Unit, Department of Psychiatry and Behavioral Neuroscience, University of Chicago, 584I South Maryland Avenue, Chicago, IL 60637, USA, Tel: + I 773834 5673, Fax: + I 773834 4536,

E-mail: rlee@yoda.bsd.uchicago.edu

Received 28 August 2015; revised 15 April 2016; accepted 18 April 2016; accepted article preview online 20 May 2016 an important step toward the development of more effective treatments.

Studies to date suggest that impulsive aggression is related to the function of brain systems mediating social and emotional behavior. On the molecular level, serotonergic (Coccaro et al, 2015), neuropeptide (Coccaro et al, 1998), and inflammatory protein abnormalities (Coccaro et al, 2014) have been linked to impulsive aggression. At the macro level, functional brain imaging studies have found evidence of exaggerated amygdala response to angry faces and diminished functional connectivity between the amygdala and orbitofrontal cortex (Coccaro et al, 2007). Impulsive aggression has been associated with abnormal patterns of task-related functional brain activity, with enhanced blood oxygen level-dependent (BOLD) activity in the amygdala and orbitofrontal cortex during provoked aggression but lower parietal activity in the medial parietal regions (New et al, 2009); enhanced amygdala BOLD reactivity to neutral faces (Bobes et al, 2013); lower striatal metabolic activity in both provoked and nonprovoked aggression as measured by FDG-PET in males (Perez-Rodriguez et al, 2012); and higher error-related dorsolateral prefrontal cortex BOLD activity (Moeller et al, 2014).

Thus, evidence to date implicates dysfunction in neural circuits mediating emotional behavior and executive 
function that rely on brain networks with hubs in both the cortical and subcortical structures.

Given that brain function depends on coordinated activity across connected brain structures, white matter connections in the brain are of potential importance in impulsive aggression. In IED, cognitive deficits appear to be circumscribed to affective and social domains but are relative, not absolute deficits of function in these domains (Best et al, 2002) and are not associated with lower intelligence (Best et al, 2002). These findings suggest that the underlying neural abnormality is more subtle than that encountered in disconnection syndromes caused by gross lesions or neurodevelopmental disorders. A more sensitive measure of white matter tract health is magnetic resonance diffusion tensor imaging (DTI) that provides estimates of white matter integrity by measuring the diffusion of water molecules (Basser and Pierpaoli, 1996; LeBihan et al, 2001). Fractional anisotropy (FA) is a scalar measure of the degree to which the diffusion process of water is anisotropic: a perfectly spherical diffusion tensor would have an FA of zero (first reported in Basser and Pierpaoli 1996; reviewed in Alexander et al, 2007).

In order to test the hypothesis that IED is associated with region-specific abnormalities in white matter integrity, a cross-sectional study was undertaken to compare white matter diffusion anisotropy between IED and control subjects using tract-based spatial statistics (TBSS) (Smith et al, 2006). To test the specificity of purported white matter integrity differences, a psychiatric control group (PC) was included in comparisons.

\section{MATERIALS AND METHODS}

\section{Subjects}

All subjects provided written, informed consent using documents approved by the Committee for the Protection of Human Subjects (IRB). A total of 132 physically healthy, right-handed subjects between the ages of 18 and 55 years participated in this study. This included 42 subjects with IED, 40 normal controls, and 50 clinical psychiatric controls with non-IED psychiatric disorders. Subjects were recruited through public service announcements seeking out individuals who had difficulty managing anger and/or aggression as well as individuals who identified themselves as healthy. Subjects with a life history of bipolar disorder, schizophrenia (or other psychotic disorder), developmental delay, or current substance dependence disorder were excluded from this study. Medical screening was conducted with a comprehensive medical history, physical and neurological examination, and drug screen for amphetamine, barbiturates, benzodiazepines, cocaine, opiates, methadone, methamphetamine, phencyclidine, oxycodone, and marijuana. Drug screening and alcohol breathalyzer was repeated before the research scan.

\section{Diagnostic Assessment}

Axis I and Axis II Personality Disorder diagnoses were made according to DSM-IV criteria (APA, 1994). The diagnosis of IED was made by Integrated Research Criteria as previously described (IED-IR: Coccaro, 2011, 2012). Diagnoses were

assessed and assigned through a best estimate process as described in previous reports (Coccaro, 2012).

\section{Treatment Characteristic of the Sample}

No subject was taking any medical or psychotropic agent for at least 4 weeks at the time of study and only 25 (19\%) of the subjects (all PC/IED) had any lifetime exposure to psychotropic agents. Of these subjects, 19 had been exposed to antidepressants, 5 to benzodiazepines, 3 to stimulants, and 2 each to antipsychotic, mood stabilizing, and sedativehypnotic agents. History of lifetime exposure to psychotropic medication had no effect on FA values $(\mathrm{F}(1,130)=0.31$, $p=0.58)$. The same proportion had a history of formal psychiatric treatment and this variable also had no effect on FA values $(F(1,130)=0.03, p=0.88)$. Subjects were also informed that initial and follow-up urine toxicology would be performed randomly just before study; illicit drug use was not detected in any subject reported herein.

\section{Assessment of Aggression and Impulsivity}

Aggression measures included the Aggression score from the Life History of Aggression assessment (LHA; Coccaro et al, 1997) and the 'physical' and 'verbal' aggression scores from the Buss-Perry Aggression Questionnaire (BPAQ; Buss and Perry, 1992). LHA aggression reflects a subject's history of actual aggressive behavior, whereas BPAQ aggression reflects a subject's self-assessment of his or her tendency to be aggressive in given situations. Impulsivity measures included the Impulsiveness Scale from the Life History of Impulsive Behavior assessment (LHIB, Coccaro and Schmidt-Kaplan, 2012) and the Barratt Impulsiveness Scale-Version 11 (BIS-11; Patton et al, 1995). LHIB impulsivity reflects a subject's history of actual impulsive behavior, whereas BIS-11 impulsivity reflects a subject's assessment of his or her tendency to be impulsive. History of suicidal behavior was assessed during the diagnostic work-up as previously described (Coccaro, 2012).

\section{Measures of Relevant Measures of Personality}

General temperament was assessed using the Neuroticism, Psychoticism, and Extraversion scales from the Eysenck Personality Questionnaire (EPQ). Psychopathic personality traits were assessed using the Screening Version of the Psychopathy Checklist (PCL-SV; Hare et al, 2003). The PCLSV has separate scores for the Callous/Unemotional (Part 1), and for the Socially Deviant (Part 2), psychopathic traits. Current global psychosocial function of subjects was assessed by the Global Assessment of Function scale (GAF, APA, 1994).

\section{Image Acquisition}

Structural and DTI data were collected with a 3T Philips MRI scanner (Best, The Netherlands). High-resolution T1-weighted anatomical data were obtained with a 3D magnetization-prepared rapid acquisition gradient-echo (MP-RAGE) sequence with the following parameters: $\mathrm{TE}=3.2 \mathrm{~ms}, \mathrm{TR}=8 \mathrm{~ms}$, preparation time $=725 \mathrm{~ms}$, flip angle $6^{\circ}$, field of view $25 \mathrm{~cm} \times 25 \mathrm{~cm}, 301$ sagittal slices, $0.6 \mathrm{~mm}$ slice thickness, and $240 \times 240$ image matrix. Turboprop-DTI 
Table I Demographic and Diagnostic Information

\begin{tabular}{|c|c|c|c|c|}
\hline & $\begin{array}{l}\text { Healthy } \\
\text { controls } \\
(N=40)\end{array}$ & $\begin{array}{l}\text { Psychiatric } \\
\text { controls } \\
(n=50)\end{array}$ & $\begin{array}{c}\text { Intermittent } \\
\text { explosive } \\
\text { disorder }(N=42)\end{array}$ & $P$-value \\
\hline Age (years) & $30.2 \pm 7.9$ & $31.0 \pm 8.7$ & $34.3 \pm 9.0$ & $=0.07^{\mathrm{a}}$ \\
\hline Gender (M/F) & $13 / 17$ & $25 / 25$ & $22 / 22$ & $N S^{b}$ \\
\hline Race (White/Non-White) & $30 / 10$ & $39 / 11$ & $17 / 25$ & $<0.001^{\mathrm{b}}$ \\
\hline SES class (I/IIIIVN ) & $3 / 33 / 3$ & $16 / 32 / 2$ & $3 / 35 / 4$ & $=0.008^{\mathrm{b}}$ \\
\hline GAF score & $83.25 \pm 4.9$ & $64.20 \pm 10.9$ & $55.58 \pm 8.4$ & $<0.001^{c}$ \\
\hline $\begin{array}{l}\text { Ever received outpatient } \\
\text { treatment }\end{array}$ & 7 & 26 & 26 & $0.177^{\mathrm{d}}$ \\
\hline Ever hospitalized & 0 & 4 & 10 & $0.576^{\mathrm{d}}$ \\
\hline MDD & & 0 & 7 & $=0.003$ \\
\hline PTSD & & । & 3 & $=0.228$ \\
\hline Panic & & 2 & 0 & $=0.190$ \\
\hline GAD & & 4 & 0 & $=0.061$ \\
\hline Antisocial & & C & 8 & $<0.001$ \\
\hline Borderline & & 4 & 18 & $<0.001$ \\
\hline Narcissistic & & । & 6 & 0.02 \\
\hline Histrionic & & I & 2 & 0.21 \\
\hline Avoidant & & 21 & 4 & 0.001 \\
\hline Dependent & & & 2 & \\
\hline OCPD & & 7 & 9 & 0.17 \\
\hline PD NOS & & 7 & 12 & 0.112 \\
\hline Mean PCL-SV & $1.23 \pm 1.6$ & $5.7 \pm 6.1$ & $8.36 \pm 5.6$ & $<0.001^{\mathrm{e}}$ \\
\hline
\end{tabular}

aSignificant after ANOVA. The post hoc test (Tukey's HSD: $p<0.05)$ for age:

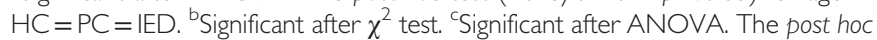
tests reveal IED $>P C>H C$. ${ }^{d}$ Nonsignificant after $\chi^{2}$ comparing the IED with

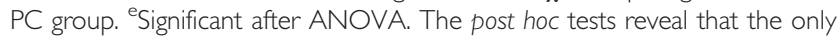
significant differences are IED $>\mathrm{HC}$ and $\mathrm{PC}>\mathrm{HC}$.

has reduced sensitivity to image artifacts from eddy currents and susceptibility-related distortions (Pipe and Zwart, 2006; Arfanakis et al, 2005). The parameters for Turboprop-DTI were: $\mathrm{TR}=4000 \mathrm{~ms}$, six spin-echoes per TR $(\mathrm{ETL}=6)$, five $\mathrm{k}$-space lines per spin-echo, 120 samples per line, 12 blades per image, field of view $24 \mathrm{~cm} \times 24 \mathrm{~cm}, 40$ axial slices, $3 \mathrm{~mm}$ slice thickness, $256 \times 256$ reconstructed image matrix, $\mathrm{b}=750 \mathrm{~s} / \mathrm{mm}^{2}$ for a set of 15 diffusion directions uniformly distributed in $3 \mathrm{D}$ space, one $\mathrm{b}=0 \mathrm{~s} / \mathrm{mm}^{2}$ image volume, and scan time of $17 \mathrm{~min}$.

\section{Image Processing}

For each subject, the brain images were extracted from the raw DTI data. The diffusion-weighted image volumes were averaged, and a mean diffusion-weighted volume was generated. The mean diffusion-weighted volume was then registered to the $b=0 \mathrm{~s} / \mathrm{mm}^{2}$ volume using $3 \mathrm{D}$ rigidbody registration. Each of the diffusion-weighted image volumes was then registered to the new mean diffusionweighted volume, using rigid-body registration, to eliminate any mismatch because of bulk motion. Eddy current and susceptibility-related distortions are not present in Turboprop-DTI data and therefore no correction was necessary. The B-matrix was appropriately reoriented. The diffusion tensor in each voxel was estimated using nonlinear tensor fitting, and maps of the FA and trace of the diffusion tensor were produced using TORTOISE (http://www.tortoi sedti.org).

\section{Statistical Analysis}

TBSS was used to compare FA values across groups. Because the location and course of white matter tracts differ between individuals, there is a need to co-register FA maps across individuals to permit voxelwise group comparisons of FA (Smith et al, 2006). The high contrast between white and gray matter FA makes minimizing the effects of any residual misregistration of white matter tract FA maps essential in the study of slender white matter tracts. TBSS addresses this issue by projecting the highest FA information from each individual's white matter tracts onto a standardized white matter skeleton. Following this process, FA maps from all subjects were first nonlinearly spatially transformed to a template, and the local FA maxima from each subject were then projected onto the white matter skeleton, thereby reducing the effects of residual misregistration (Smith et al, 2006).

Linear regression models were used to test for differences in FA values across groups, controlling for age, sex, lifetime alcohol dependence, and any nonalcohol drug dependence. Linear regression was used to test for differences in the trace of the diffusion tensor across groups. The null distribution was built using the "randomise" tool in FSL (FMRIB, University of Oxford, UK) and 5000 permutations of the data (Winkler et al, 2014). Differences were considered significant at $p<0.05$, familywise error (FWE) corrected. The Threshold-Free Cluster Enhancement (TFCE) method was used to define clusters with significant differences (Smith and Nichols, 2009).

The mean FA and trace values in clusters that showed significant differences across groups were extracted for each subject. Comparisons of regional values across groups were performed by $t$-test, with correction for unequal variances where necessary, analysis of covariance (ANCOVA), and by the $\chi^{2}$ tests. Correlational analyses of regional tensor-derived values with behavioral variables were conducted including Pearson's correlation, partial correlation, and multiple regression. A two-tailed $\alpha$-value of 0.05 was used to denote statistical significance, uncorrected for multiple comparisons. Composite variables for 'aggression,' 'impulsivity,' and 'impulsive aggression' were created in a data-reduction step by taking the average of each subject's $z$-scores for the primary behavioral measures, as described in Coccaro et al (2010).

\section{RESULTS}

The demographic characteristics are displayed in Table 1. These groups did not differ in age or distribution in gender but did differ in distribution in race and Hollinghead's socioeconomic (SES) class. The difference in race was due to more non-whites among the IED vs the HC/PC groups and to more SES class I subjects among the PC vs the NC/IED subjects.

\section{Tract-Based Spatial Statistics Results}

TBSS analysis revealed significantly lower FA values in IED patients than healthy controls in two clusters within the superior longitudinal fasciculus (SLF). These two clusters were located in the SLF I and SLF II subdivisions (Figure 1) (Makris et al, 2005). No other parts of the brain showed significant FA or trace differences across groups. Mean FA 


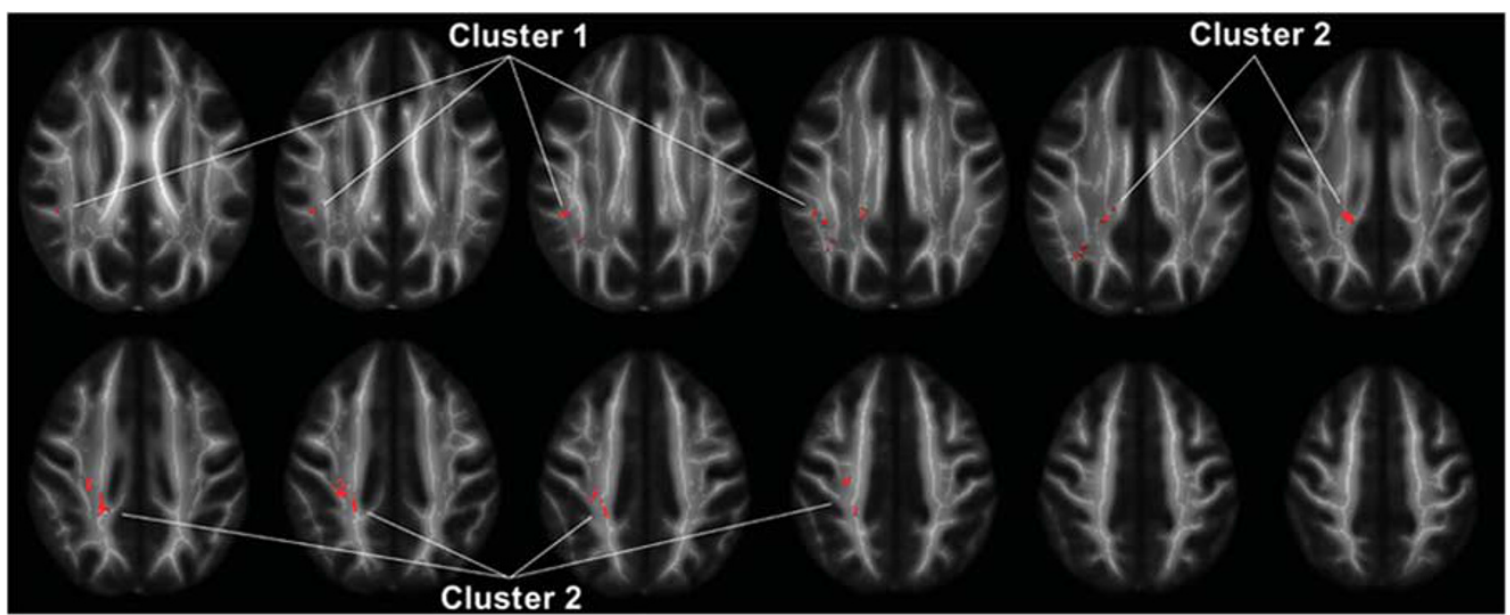

Figure I Regions of the white matter skeleton in which FA in IED subjects was significantly lower than healthy controls ( $p<0.05$, corrected for multiple comparisons). The location of clusters I and 2 used in the region-of-interest analysis is shown. Template FA maps and the white matter skeleton used in the analysis are shown in the background in grayscale.

values from these two clusters followed a normal distribution, and were highly correlated $(r=0.59, n=132, p<0.001)$.

\section{FA of Clusters 1 and 2 as a Function of Subject Group and Covariates}

Multiple regression analysis revealed that among all demographics variables, only gender $(B=0.023 \pm 0.009, \beta=0.216$, $\left.t_{127}=2.51, p=0.013\right)$ and SES class $(\mathrm{B}=0.025 \pm 0.010$, $\left.\beta=0.221, t_{127}=2.60, p=0.010\right)$ correlated significantly with regional FA values, with female gender and higher SES class associated with higher FA values. Accordingly, these two variables were used as covariates in the analyses reported below. One-way ANOVA of the FA of cluster 1 (Figure 1) revealed a significant effect of group $(\mathrm{F}(2,129)=9.9$, $p<0.001)$. The post hoc testing with Tukey's method revealed that IED $<$ PC $(p=0.03)$ and HC $(p<0.001)$ but PC and HC were not significantly different. Adding gender and SES to the model did not significantly change the results. One-way ANOVA of the FA of cluster 2 (Figure 1) revealed a significant effect of group $(\mathrm{F}(2,129)=10.35, p<0.001)$. The post hoc testing with Tukey's method revealed that IED $<$ PC $(p=0.03)$ and HC $(p<0.001)$ and PC $<$ HC at a trend level of significance $(p=0.07)$. Adding gender and SES to the model did not significantly change the results.

\section{FA and Impulsive Aggression}

Across all subjects, a significant inverse correlation was observed between FA value of cluster 1 and composite impulsive aggression $(r=-0.428, n=76, p<0.001$; Figure 2$)$, composite aggression $(r=-0.415, n=76, p<0.001)$, and composite impulsivity $(r=-0.331, n=76, p<0.001)$. Partial correlation, controlling for SES and sex, did not meaningfully affect these relationships (composite impulsive aggression: $r=-0.346, \mathrm{df}=72, \quad p<0.001$; composite aggression: $r=$ $-0.418, \mathrm{df}=72, p=0.004$; composite impulsivity: $r=-0.332$, $\mathrm{df}=73, p<0.001)$. A similar pattern was seen with correlations of the FA values of cluster 2 with composite impulsive aggression ( $r=-0.346, n=76, p=0.002$; Figure 3$)$, composite aggression $(r=-0.305, n=76, p=0.007)$, and composite impulsivity ( $r=-0.297, n=76, p=0.009$ ). Partial correlation, controlling for SES and sex, did not meaningfully affect these relationships (composite impulsive aggression: $r=-0.337$, $\mathrm{df}=72, p=0.003$; composite aggression: $r=-0.294, \mathrm{df}=72$, $p=0.01$; composite impulsivity: $r=-0.297, \mathrm{df}=73, p=0.01$ ).

Multiple regression analysis of the FA value of cluster 1 with the regressors PCL-SV 'callous/unemotional' traits and PCL-SV 'socially deviant' traits and sex and SES as covariates did not result in a significant model $(\mathrm{F}(4,63)=1.5$, $p=0.212$ ). Similarly, multiple regression analysis of cluster 2 FA values did not result in a significant model $(\mathrm{F}(4,63)=2.268, p=0.07)$. These results suggest that individual differences in cluster 1 and cluster 2 FA values were not significantly explained by psychopathy traits.

\section{FA Values and History of Self-Directed Aggression in the PC/IED Group}

Among the PC/IED group, 14 (16\%) had a life history of a suicide attempt $(\mathrm{SA}+)$ and $8(9 \%)$ had a life history of self-injurious behavior (SIB). For cluster 1, FA values were significantly lower among SA+ subjects controlling for SES and sex (SA+: $0.536 \pm 0.053$ vs SA - : $0.573 \pm 0.052$, $\mathrm{F}(1,127)=5.183, p=0.02)$. For cluster 2 , FA values were not significantly different (SA+: $0.511 \pm 0.056$ vs SA-: $0.544 \pm 0.068, F(1,127)=2.531, p=0.114)$. FA for clusters 1 and 2 did not differ as a function of a history of SIB, controlling for SES and sex (cluster 1: SIB $+=0.528 \pm 0.067$ vs SIB $-=0.545 \pm 0.051, \mathrm{~F}(1,127)=2.369, p=0.126$; cluster $2: \mathrm{SIB}+=0.519 \pm 0.072$ vs SIB $-: 0.541 \pm 0.067, \mathrm{~F}(1,127)=0.459$, $p=0.5$ ).

\section{FA Values and Major Depressive Disorder (MDD)}

ANCOVA of cluster 1 FA values for MDD covarying for current IED, sex, and SES did not find a significant association of MDD with significantly $\mathrm{FA}$ value $(\mathrm{F}(1,126)=1.693, \quad p=0.196$ respectively). ANCOVA of cluster 2 FA values for MDD similarly did not find a 


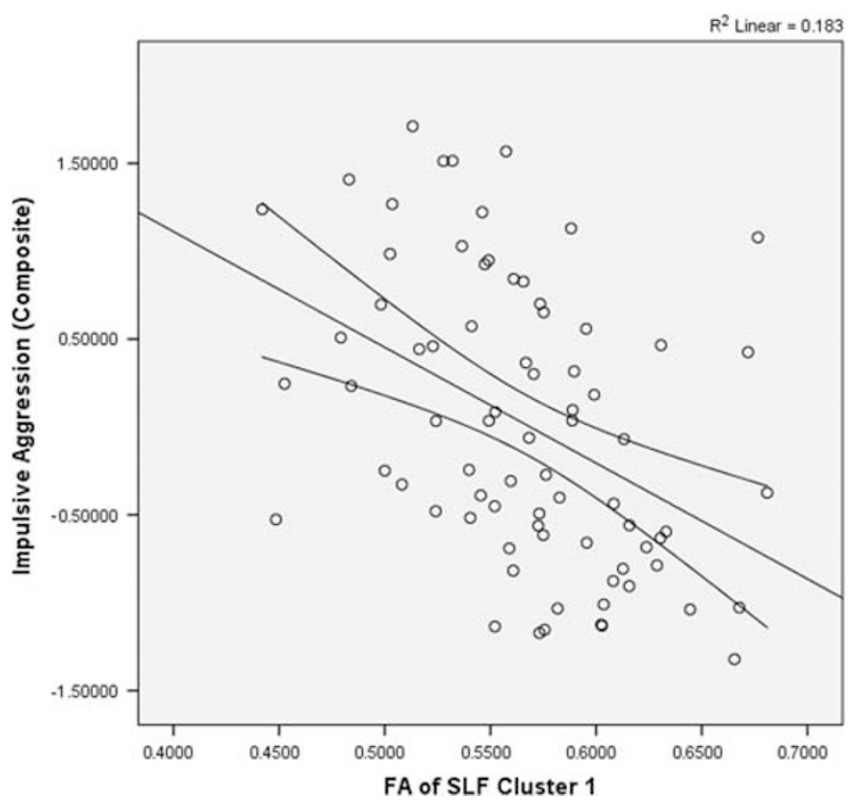

Figure 2 Scatterplot of mean FA of SLF cluster I vs impulsive aggression. The mean FA value of cluster I in the SLF was significantly and inversely correlated with composite aggression score $(r=-0.428, n=76, p<0.00 \mathrm{I})$.

significant association with $\mathrm{FA}$ values $(\mathrm{F}(1,127)=0.239$, $p=0.91$ ). Thus, history of MDD did not account for the group differences between IED and HC research participants.

\section{FA Values and Personality Disorders}

Four personality disorders (borderline, antisocial, narcissistic, and avoidant) with sufficient representation in the sample were assessed for their relationship to FA using multivariate-ANCOVA. The four personality disorders were distributed unequally across the PC and IED groups (see Table 1). MANCOVA of cluster $1 \mathrm{FA}$ values for these four personality disorders, covarying for current IED, sex, and SES, revealed that only borderline personality disorder was associated with significantly decreased FA values $(\mathrm{F}(1,117)=5.462, p=0.02)$. No interactions were significant. MANCOVA of cluster $2 \mathrm{FA}$ values for these factors and covariates revealed that only avoidant personality disorder was associated with decreased $\mathrm{FA}$ values $(\mathrm{F}(1,117)=4.389$, $p=0.04)$. No interactions were significant.

\section{DISCUSSION}

Evidence was found for altered white matter integrity as measured by FA in IED relative to normal control subjects in the SLF. This is the first report linking white matter integrity to adults with IED. The approach taken used TBSS and a hypothesis-free approach with correction for multiple comparisons.

Although we are not aware of any other DTI studies in adults with IED, DTI studies have been conducted in the populations with the related conditions of borderline personality disorder, antisocial personality disorder, and

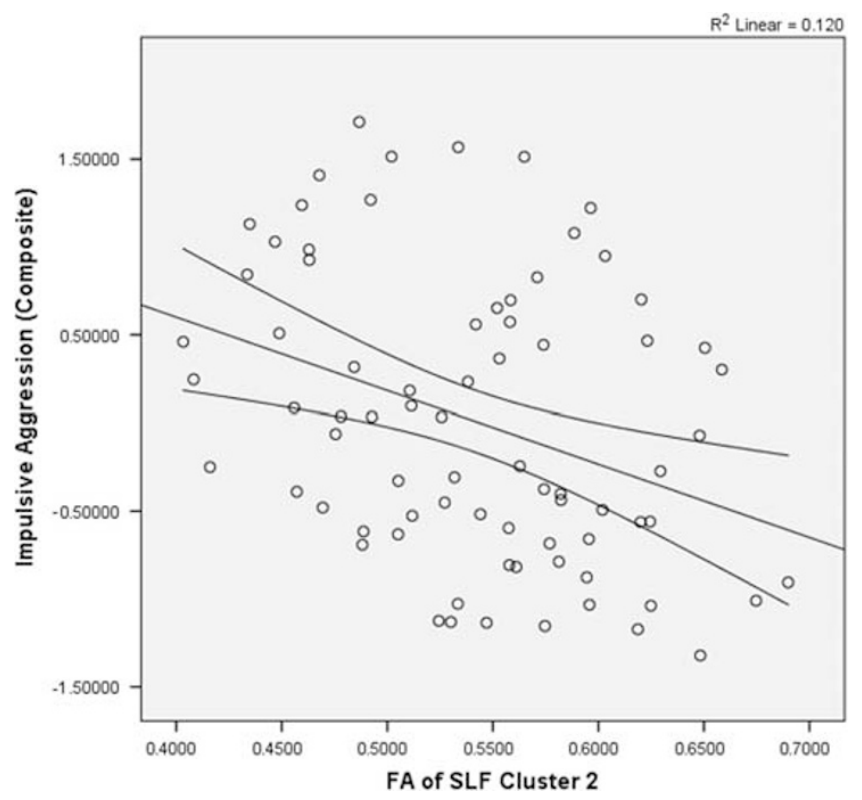

Figure 3 Scatterplot of mean FA of SLF cluster 2 vs impulsive aggression. The mean FA value of cluster 2 in the SLF was significantly and inversely correlated with composite aggression score $(r=-0.346, n=76, p=0.002)$.

conduct disorder. All three of these conditions are characterized by problematic impulsivity.

BPD in adults has been associated with decreased FA in the corpus callosum and white matter of the orbitofrontal cortical regions (voxelwise analysis; Carrasco et al, 2012), the corpus callosum and the dorsal anterior cingulate (Rüsch et al, 2007), and the cingulum and fornix (Whalley et al, 2015). Two studies in adolescents with BPD found evidence for decreased white matter integrity in the inferior longitudinal fasciculus in adolescents with BPD (not found in adults with BPD in this study; New et al, 2013), the SLF, and fornix (Maier-Hein et al, 2014). Our findings of decreased FA in the SLF in IED as well as BPD is most similar to the results found in adolescents with BPD.

In the case of antisocial personality disorder and the related condition of conduct disorder, a somewhat similar pattern of results has been reported. Criminal psychopathy has previously been associated with decreased FA of the uncinate fasciculus, the white matter track connecting the amygdala to prefrontal cortex (Craig et al, 2009). A wholebrain study of adults with ASPD similarly found decreased FA in the uncinated fasciculus (Sundram et al, 2012), as well as the genu of the corpus callosum, inferior fronto-occipital fasciculus, anterior corona radiata, and the internal capsule. In conduct disorder, an ROI-based study (Zhang et al, 2014) found higher FA in the uncinate fasciculus in boys. A single study has examined the relationship between aggression in children with ADHD and white matter connectivity between the frontal lobes and nucleus accumbens, using probabilistic tractography (Cha et al, 2015). This study found evidence for decreased frontal-accumbal white matter connectivity in aggressive children with ADHD. These disparate findings have not yet been replicated and remain preliminary. However, the lack of overlap of DTI findings between psychopathic traits and IED suggest that at the very least, our white matter findings are not driven exclusively 
psychopathic traits. Future studies should be conducted to examine the extent to which white matter FA is differentially related to hostile versus instrumental aggression.

The finding of white matter abnormalities in the SLF in IED is somewhat unexpected but intriguing. The SLF connects parietotemporal association areas with the frontal lobe. Given the importance of the inferior parietal and posterior temporal cortex in social cognition (Decety and Lamm, 2007; Young et al, 2010), and evidence for disturbed social cognition in IED (Coccaro et al, 2009), findings of disrupted white matter connectivity in these tracts in IED could be of considerable importance. First described by Theodore Meynert (Wernicke, 1874), lesioning of the SLF was proposed by Norman Geschwind to cause a set of disconnection syndromes, characterized by impairments in language and praxis (Geschwind, 1965a,b; Catani and Ffytche, 2005). The SLF can be divided into four subcomponents (Makris et al, 2005). SLF I, II, and III connect the frontal lobes to the medial and superior parietal regions, the caudal inferior parietal lobe, and rostral inferior parietal lobule respectively. The arcuate fasciculus links the caudal superior temporal gyrus to Broca's area. These patterns of connectivity would suggest that the SLF has a role in the executive functions of motor planning, visuospatial working memory, and language; this is confirmed by reports of association of SLF white matter integrity with measures of executive function (Sasson et al, 2013). Other psychiatric disorders that have been associated with abnormal indices of SLF white matter integrity include anorexia nervosa (Via et al, 2014; Frieling et al, 2012) and borderline personality disorder (Maier-Hein et al, 2014). Our finding of decreased FA in SLF I and II indicates impaired connectivity between the frontal lobes and medial, superior, and caudal inferior parietal lobes. The cause of lower FA in the IED subjects cannot be identified from this cross-sectional study. Previous work has found that FA of the SLF is moderately heritable (Blokland et al, 2012), suggesting a role for genetic factors.

In summary, we provide for the first time evidence for subtle disruption of the long-range white matter tracks connecting the parietal and temporal lobes to the frontal cortex in IED. These disruptions have some specificity for IED, as FA in the regions of the SLF identified with voxelwise analysis is lower in IED compared with psychiatric controls.

\section{FUNDING AND DISCLOSURE}

The research was partially funded by 1R21MH083309 (PI: Royce Lee). The authors declare no conflict of interest.

\section{ACKNOWLEDGMENTS}

Special thanks are given to Mike Angstadt and Rosemary McCarron who were involved in processing of MRI and DTI data, and to Margaret Wieczorek for management of the research staff. Dr Lee has received research funding from NIMH and Azevan Pharmaceuticals. Dr Keedy has received research funding from NIMH. Dr Arfanakis has received research funding from NIH and the Alzheimer's Assocation. Dr Coccaro has received research funding from NIMH and is on scientific board of advisors for Azevan Pharmaceuticals.

\section{REFERENCES}

Alexander AL, Lee JE, Lazar M, Field AS (2007). Diffusion tensor imaging of the brain. Neurotherapeutics 4: 316-329.

American Psychiatric Association (1994). Diagnostic and Statistical Manual, 4th edn (DSM-IV). American Psychiatric Press: Washington, DC.

Arfanakis K, Gui M, Lazar M (2005). White matter tractography by means of Turboprop diffusion tensorimaging. Ann NY Acad Sci 1064: 78-87.

Basser PJ, Pierpaoli C (1996). Microstructural and physiological features of tissues elucidated by quantitative-diffusiontensor MRI. J Magn Reson B 111: 209-219.

Best M, Williams JM, Coccaro EF (2002). Evidence for a dysfunction prefrontal circuit in patients with impulsive aggressive disorder. Proc Natl Acad Sci USA 99: 8448-8453.

Blokland GA, de Zuicaray GI, McMahon KL, Wright MJ (2012). Genetic and environmental influences on neuroimaging phenotypes: a meta-analytical perspective on twin imaging studies. Twin Res Hum Genet 15: 351-371.

Bobes MA, Ostrosky F, Diaz K, Romero C, Borja K, Santos Y et al (2013). Linkage of functional and structural anomalies in the left amygdala of reactive-aggressive men. Soc Cogn Affect Neurosci 8: 928-936.

Buss AH, Perry M (1992). The aggression questionnaire. J Pers Soc Psychol 63: 452-459.

Carrasco JL, Tajima-Pozo K, Diaz-Marsa M, Casado A, Lopez-Ibo JJ, Arrazola J et al (2012). Microstructural white matter damage at orbitofrontal areas in borderline personality disorder. J Affect Disord 139: 149-153.

Catani M, ffytche DH (2005). The rises and falls of disconnection syndromes. Brain 128: 224-2239.

Cha J, Fekete T, Siciliano F, Biezonski D, Greenhill L, Pliszka SR et al (2015). Neural correlates of aggression in medication naïve children with ADHD: multivariate analysis of morphometry and tractography. Neuropsychopharmacology 40: 1717-1725.

Coccaro EF (2011). Intermittent explosive disorder: development of integrated research criteria for Diagnostic and Statistical ManualFifth Edition. Compr Psychiatry 52: 119-125.

Coccaro EF (2012). Intermittent explosive disorder as a disorder of impulsive aggression for DSM-5. Am J Psychiatry 169: 577-588.

Coccaro EF, Berman ME, Kavoussi RJ (1997). Assessment of life history of aggression: development and psychometric characteristics. Psychiatry Res 73: 147-157.

Coccaro EF, Fanning JR, Phan KL, Lee R (2015). Serotonin and impulsive aggression. CNS Spectr 20: 295-302.

Cocarro EF, Kavoussi RJ, Hauger RL, Cooper TB, Ferris CF (1998). Cerebrospinal fluid vasopressin levels: correlates with aggression and serotonin function in personality-disordered subjects. Arch Gen Psychiatry 55: 708-714.

Coccaro EF, Lee R, Coussons-Read M (2014). Elevated plasma inflammatory markers in individuals with intermittent explosive disorder and correlation with aggression in humans. JAMA Psychiatry 71: 158-165.

Coccaro EF, Lee RJ, Kavoussi RJ (2009). A double-blind, randomized, placebo-controlled trial of fluoxetine in patients with intermittent explosive disorder. J Clin Psychiatry 70: 653-662.

Coccaro EF, Lee R, Kavoussi RJ (2010). Aggression, suicidality, and intermittent explosive disorder: serotonergic correlates in personality disorder and healthy control subjects. Neuropsychopharmacology 35: 435-444.

Coccaro EF, Lee R, McCloskey MS (2014). Relationship between psychopathy, aggression, anger, impulsivity, and intermittent explosive disorder. Aggress Behav 40: 526-536.

Coccaro EF, McCloskey MS, Fitzgerald DA, Phan KL (2007). Amygdala and orbitofrontal reactivity to social threat in 
individuals with impulsive aggression. Biol Psychiatry 62: $168-178$

Coccaro EF, Noblett KL, McCloskey MS (2009). Attributional and emotional responses to emotionally ambiguous cues: validation of a new assessment of social/emotional information processing in healthy adults and impulsive aggressive patients. J Psychiatr Res 49: 915-925.

Coccaro EF, Schmidt-Kaplan CA (2012). Life history of impulsive behavior: development and validation of a new questionnaire. J Psychiatr Res 46: 346-352.

Craig MC, Catani M, Deeley Q, Latham R, Daly E, Kanaan R et al (2009). Altered connections on the road to psychopathy. Mol Psychiatry 14: 946-953.

Decety J, Lamm C (2007). The role of the right temporopariental junction in social interaction: how low-level computational processes contribute to meta-cognition. Neuroscientist 13: 580-593.

Frieling H, Fischer J, Wilhelm J, Engelhorn T, Bleich S, Hillemacher T et al (2012). Microstructural abnormalities of the posterior thalamic radiation and the mediodorsal thalamic nuclei in females with anorexia nervosa-a voxel based diffusion tensor imaging (DTI) study. J Psychiatr Res 46: 1237-1242.

Geschwind N (1965a). Disconnexion syndromes in animals and man, I. Brain 88: 237-294.

Geschwind N (1965b). Disconnexion syndromes in animals and man, II. Brain 88: 585-644.

Glenn AL, Raine A (2009). Psychopathy and instrumental aggression: evolutionary, neurobiological, and legal perspectives. Int J Law Psychiatry 32: 253-258.

Hare RD. The Hare Psychopathy Checklist-Revised, 2nd edn. MultiHealth Systems: Toronto, ON, 2003.

Kessler RC, Coccaro EF, Fava M, Jaeger S, Jin R, Walters E (2006). The prevalence and correlates of DSM-IV intermittent explosive disorder in the National Comorbidity Survey Replication. Arch Gen Psychiatry 63: 669-678.

LeBihan D, Mangin JF, Poupon C, Clark CA, Pappata S, Molko N et al (2001). Diffusion tensor imaging: concepts and applications. J Magn Reson Imaging 13: 534-546.

Maier-Hein KH, Brunner R, Lutz K, Henze R, Parzer P, Feigl N et al (2014). Disorder-specific white matter alterations in adolescent borderline personality disorder. Biol Psychiatry 75: 81-88.

Makris N, Kennedy DN, McInerney S, Sorensen AG, Wang R, Caviness VS Jr et al (2005). Segmentation of subcomponents within the superior longitudinal fascicle in humans: a quantitative, in vivo, DT-MRI study. Cereb Cortex 15: 854-869.

McCloskey MS, Noblett KL, Deffenbacher JL, Gollan JK, Coccaro EF (2008). Cognitive-behavioral therapy for intermittent explosive disorder: a pilot randomized clinical trial. J Consult Clin Psychol 76: 876-886.

Moeller SJ, Froböse MI, Konova AB, Misyrlis M, Parvaz MA, Goldstein RZ et al (2014). Common and distinct neural correlates of inhibitory dysregulation: stroop fMRI study of cocaine addiction and intermittent explosive disorder. J Psychiatr Res 58: $55-62$.
New AS, Carpenter DM, Perez-Rodriguez MM, Ripoll LH, Avedon J, Patil U et al (2013). Developmental differences in diffusion tensor imaging parameters in borderline personality disorder. J Psychiatr Res 47: 1101-1109.

New AS, Hazlett EA, Newmark RE, Zhang J, Triebwasser J, Meyerson D et al (2009). Laboratory induced aggression: a positron emission tomography study of aggressive individuals with borderline personality disorder. Biol Psychiatry 66: 1107-1114.

Patton JH, Stanford MS, Barratt ES (1995). Factor structure of the Barratt impulsiveness scale. J Clin Psychology 51: 768-774.

Perez-Rodriguez MM, Hazlett EA, Rich EL, Ripoll LH, Weiner DM, Spence N et al (2012). Striatal activity in borderline personality disorder with comorbid intermittent explosive disorder: sex differences. J Psychiatr Res 46: 797-804.

Pipe JG, Zwart N (2006). Turboprop: improved PROPELLER imaging. Magn Reson Med 55: 380-385.

Rüsch N, Weber M, Il'yasov KA, Lieb K, Ebert D, Hennig J et al (2007). Inferior frontal white matter microstructure and patterns of psychopathology in women with borderline personality disorder and comorbid attention-deficit hyperactivity disorder. Neuroimage 35: 738-747.

Sasson E, Doniger GM, Pasternak O, Tarrasch R, Assaf Y (2013). White matter correlates of cognitive domains in normal aging with diffusion tensor imaging. Front Neurosci 7: 1-13.

Smith SM, Jenkinson M, Johansen-Berg H, Rueckert D, Nichols TE, Mackay CE et al (2006). Tract-based spatial statistics: voxelwise analysis of multi-subject diffusion data. Neuroimage 31: 1487-1505.

Smith SM, Nichols TE (2009). Threshold-free cluster enhancement: addressing problems of smoothing, threshold dependence and localization in cluster inference. Neuroimage 44: 83-98.

Sundram F, Deeley Q, Sarkar S, Daly E, Latham R, Craig M et al (2012). White matter microstructural abnormalities in the frontal lobe of adults with antisocial personality disorder. Cortex 48: 216-229.

Via E, Zalesky A, Sánchez I, Forcano L, Harrison BJ, Pujol J et al (2014). Disruption of brain white matter microstructure in women with anorexia nervosa. J Psychiatry Neurosci 39: 367-375.

Wernicke C (1874). Der Aphasiche Symptomenkomplex. Cohnund Weigert: Breslau.

Whalley HC, Nickson T, Pope M, Nicol K, Romaniuk L, Bastin ME et al (2015). White matter integrity and its association with affective and interpersonal symptoms in borderline personality disorder. Neuroimage 7: 476-481.

Winkler AM, Ridgway GR, Webster MA, Smith SM, Nichols TE (2014). Permutation inference for the general linear model. Neuroimage 92: 381-397.

Young L, Dodell-Feder D, Saxe R (2010). What gets the attention of the temporo-parietal junction? An fMRI investigation of attention and theory of mind. Neuropsychologia 48: 2658-2664.

Zhang J, Gao J, Shi H, Huang B, Wang X, Situ W et al (2014). Sex differences of uncinate fasciculus structural connectivity in individuals with conduct disorder. Biomed Res Int 2014: 673165. 